\title{
Optimizing dc-resistance of a foil wounded toroidal inductor combining matlab and
} comsol

Schneider, Henrik; Andersen, Thomas; Knott, Arnold; Andersen, Michael A. E.

Published in:

Proceedings of IEEE AFRICON 2013

Link to article, DOI:

10.1109/AFRCON.2013.6757712

Publication date:

2013

Link back to DTU Orbit

Citation (APA):

Schneider, H., Andersen, T., Knott, A., \& Andersen, M. A. E. (2013). Optimizing dc-resistance of a foil wounded toroidal inductor combining matlab and comsol. In Proceedings of IEEE AFRICON 2013 IEEE.

https://doi.org/10.1109/AFRCON.2013.6757712

\section{General rights}

Copyright and moral rights for the publications made accessible in the public portal are retained by the authors and/or other copyright owners and it is a condition of accessing publications that users recognise and abide by the legal requirements associated with these rights.

- Users may download and print one copy of any publication from the public portal for the purpose of private study or research.

- You may not further distribute the material or use it for any profit-making activity or commercial gain

- You may freely distribute the URL identifying the publication in the public portal 


\title{
Optimizing DC-Resistance of a Foil Wounded Toroidal Inductor Combining Matlab and Comsol
}

\author{
Department of Electrical Engineering, \\ Technical University of Denmark, \\ Kgs. Lyngby, 2800, Denmark
}

Henrik Schneider, Thomas Andersen, Arnold Knott and Michael A. E. Andersen

\begin{abstract}
- an optimization routine is presented to optimize the shape of a foil winding of a toroid inductor in terms of the DC resistance. MATLAB was used to define the geometry of the foil winding and COMSOL was used to import the geometry and create a 3D finite element model. The initial parameters, the execution and the results of the optimization routine were all managed from a graphical user interface and the feedback from COMSOL in terms of DC resistance was used to find and plot the optimal shape of the foil. The DC resistance was improvement by $31 \%$ compared with previous work for a 10 turn toroidal inductor.
\end{abstract}

Keywords-Inductor;Toroid;FEM;Foil;Optimization;GUI

\section{INTRODUCTION}

The conventional wire wound toroid is used in many power electronic applications such as EMC filters, power inductors, transformers and so on. However it has the following disadvantages regarding the winding scheme and the manufacturing/production process [1,2]:

- The spacing between the windings increases gradually from the inner diameter towards the outer diameter of the core limiting the utilization of the winding area.

- For large wire diameters a hook/pull type manufacturing machine is used. The space required for the hook and the wire being pulled through the center of the core further decrease the utilized winding area.

- For large wire diameters manual work is required for feeding the hook with the wire leading to increased cost.

- Complex manufacturing machines are needed for winding due to non-separable core.

- Manufacturer lead time and price strongly depends on the purchase history and number of ordered magnetic components.

- The leaded toroid may be placed and soldered manually in a production increasing time to market and cost.

By using thinner wires in parallel the disadvantages regarding the winding space can be improved [1]. However this work focus on a different approach using a hybrid foil + printed circuit board (PCB) based winding scheme in an

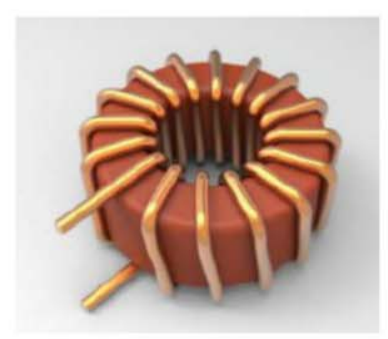

a)

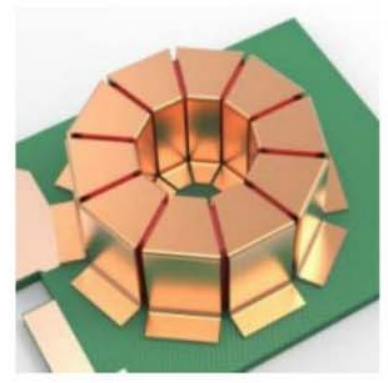

b)

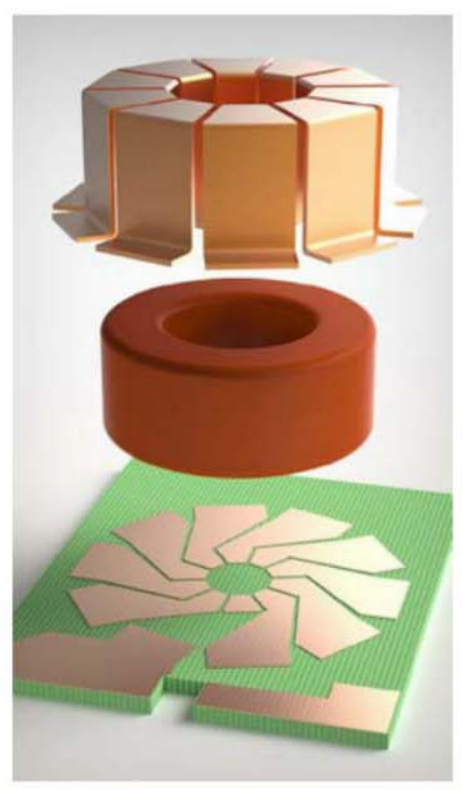

c)

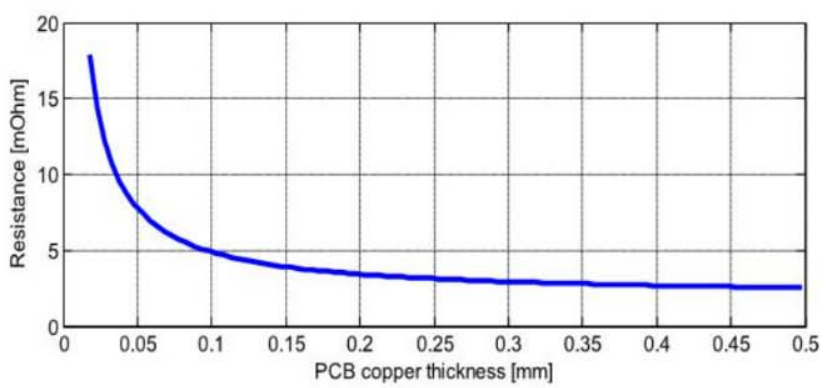

d)

Figure 1. a) Conventional toroidal wire wound inductor. b) Surface mounted hybrid inductor. c) Exploded view of a hybrid inductor. d) Example of a DC resistance vs. PCB layer thickness plot for a hybrid inductor.

attempt to improve both the utilized winding space and improve the time to market by creating more freedom in the manufacturing and production process.

The basic idea is to cut and bend copper foils into "U" shaped pieces that fit around the toroidal core as shown in Figure 1.b and Figure 1.c. The number of bended foil pieces determines the number of turns and in a final product all the foil pieces would be pre-attached to a plastic former for easy 
handling and alignment on the PCB. This assembly is referred to as the winding assembly. The foil pieces needs to be connected through the PCB traces to complete the winding. In the case of an inductor the completed inductor (winding assembly + connecting PCB tracks) is referred to as the hybrid inductor. A single patent [3] was found late in this work describing the same idea. However no commercial product or relevant publications have been found on the subject.

Different arrangements of the winding assembly with different foil thicknesses, number of turns and core dimensions for various purposes could be sold via component distributors all over the world. Due to large scale production and distributed stock of winding assemblies this could reduce cost and time to market. In addition a fully automatic production process could be utilized where the core and winding assembly were placed by pick and place machinery and soldered along with other surface mount devices (SMD's).

In previous work [4] a hybrid inductor with 15 turns, a foil thickness of 500um and a single layer PCB with a layer thickness of $70 \mathrm{um}$ was implemented as a prototype. The measurements were used to evaluate a 3D Finite element model (FEM) created in COMSOL with good agreement. The DC resistance of a 15 turn hybrid inductor with a foil thickness of 500um was simulated and plotted vs. varying PCB layer thicknesses as shown in fig. 1.d. The results showed that typical available PCB layer thicknesses (18um $210 \mathrm{um}$ ) are a bottle neck for the DC resistance in a hybrid inductor.

In the implemented model the bended copper pieces went straight over the core and the traces in the PCB was angled to complete the winding as shown in Figure 1.a. and Figure 1.b. It is easy to imagine how the DC resistance would be improved if the thin traces in the PCB were straight and short and the thicker copper pieces were angled over the core connecting the winding. It is however hard to predict the optimum angles of each segment in a turn and to predict the impact on the DC resistance. It is necessary to find an answer to these questions in order to take full advantage of the hybrid inductor.

\section{GEOMETRY}

In order to find the optimum shape of the foil winding a set of equations governing the geometry of a bendable foil around a toroid was setup in MATLAB. The initial parameters used to define and draw the foil shape was the core dimension, number of turns, clearance between the turns, starting position of the winding following the tangent of the core, angle of each segment in a turn and the thickness of each segment in a turn.

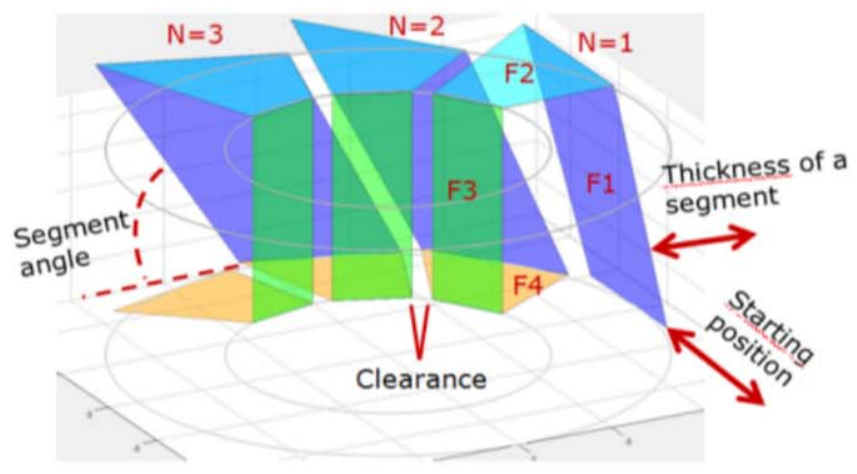

Figure 2. Geometry of the winding and the related parameters

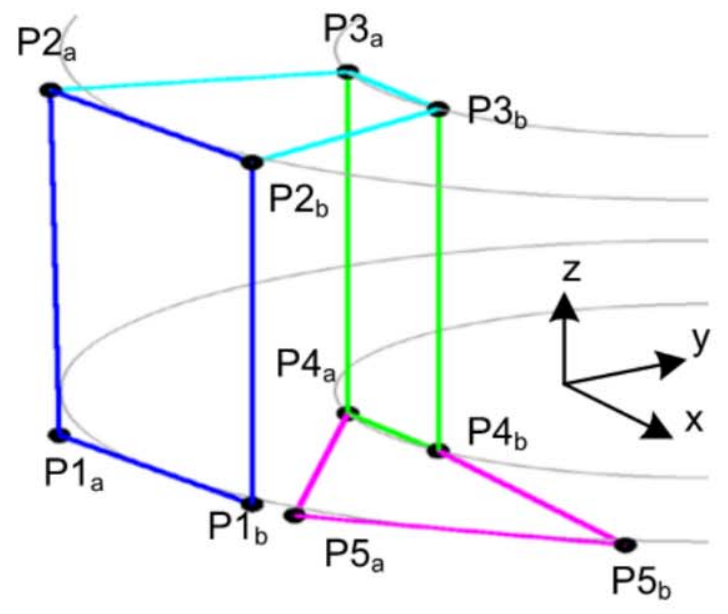

Figure 3. Geometry of the winding and the related parameters

A graphical representation of these parameters is shown in Figure 2. The four segments of a turn are labeled F1, F2, F3 and F4.

\section{A. Procedure of geometry}

The bendable turn is based on 10 points, connecting the point by straight lines forms four faces as shown in Figure 3. For a winding to be continuous point P5a and P5b must equal point $\mathrm{P} 1 \mathrm{a}$ and $\mathrm{P} 1 \mathrm{~b}$ rotated the angle of a wingding space. The winding space angle is given by (1) where $\mathrm{N}$ is the number of turns.

$$
A_{w s}=\frac{2 \pi}{N}
$$

The position of point $\mathrm{Pla}$ is defined on the tangent line $\left(\mathrm{M}_{\text {tangent }}\right)$ by a starting position angle $\left(\mathrm{A}_{\mathrm{sp}}\right)$. This is illustrated on Figure 4. P2a is based on the location of P1a and rotated according to its predefined segment angle. Likewise is P3a defined by the position of $\mathrm{P} 2 \mathrm{a}$. For the turn to be bendable P4a is based on the interception with the line formed between P3a and $\mathrm{P} 4 \mathrm{~b}$ in the $\mathrm{x}-\mathrm{y}$ plan. The remaining "b"-points are calculated from there respected "a"-points by rotating a winding space angle subtracted the distance of clearance.

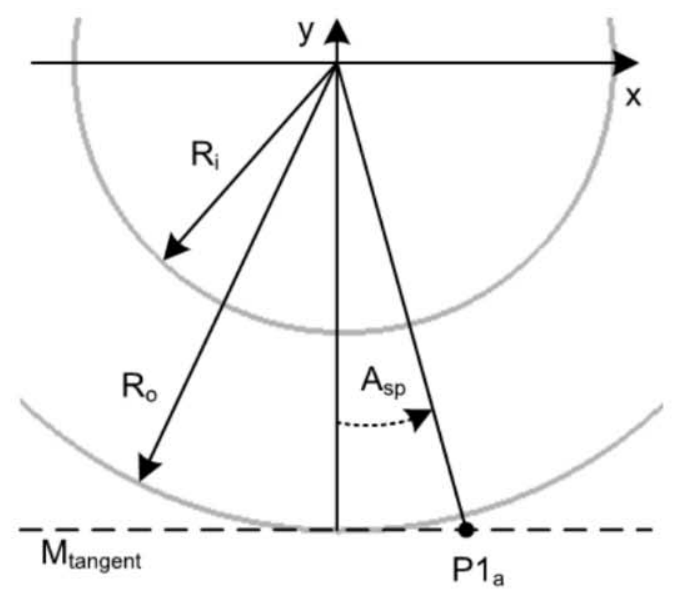

Figure 4: Definition of starting position for point $\mathrm{P} 1_{3}$ 


\section{B. Illustation}

In Figure 5.a the starting position is offset to the left of the core center line. In Figure 5.b-e the angle of the individual segments of a single turn is varied with a non offset starting position. At first the bottom layer (F4) is angled $100 \%$ which indicates that all the other segments in the turn are not angled. Then the remaining segments are angled $100 \%$ one at a time. In Figure 5.f the number of turns is very low to verify the

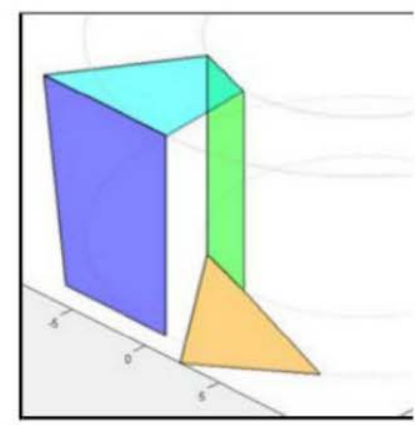

a)

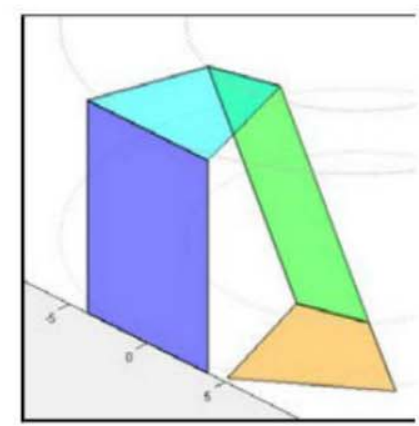

c)

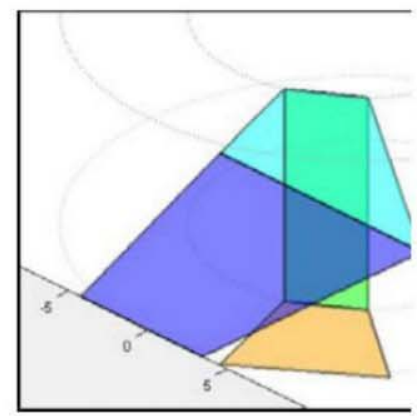

e)

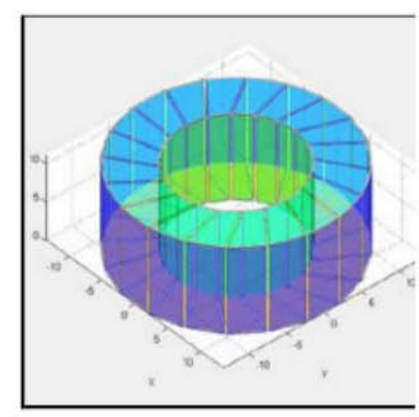

g)

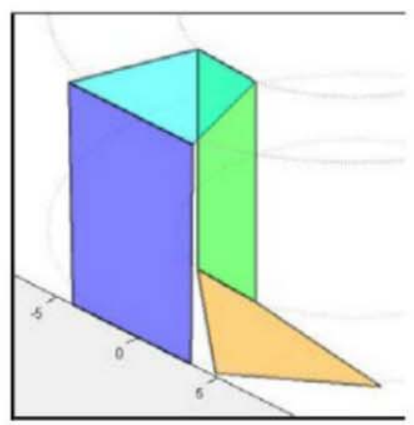

b)

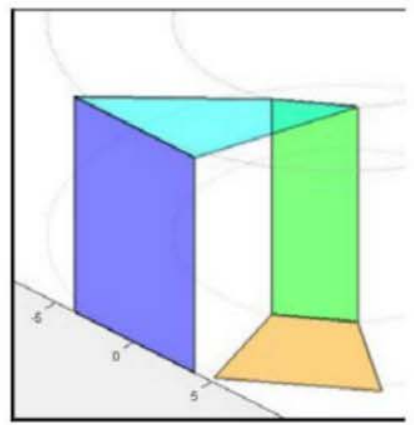

d)

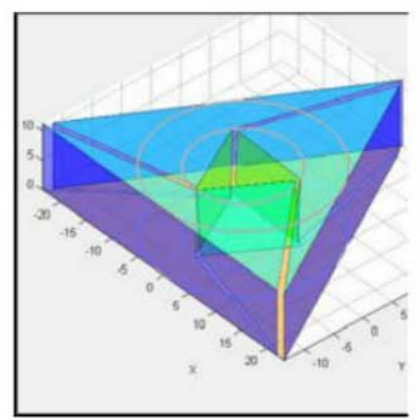

f)

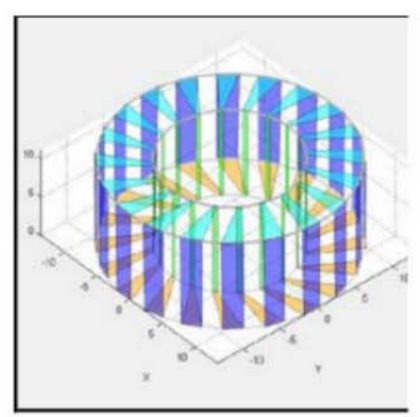

h)
Figure 5. 3D plots generated using the GUI. a) Full bottom (F4) twist and left shifted start position. b) Full bottom (F4) twist and centered start position. c) Full inner (F3) twist. d) Full top (F2) twist. e) Full outer (F1) twist. f) A few number of turns. g) Many number of turns. h) Increased clearance.

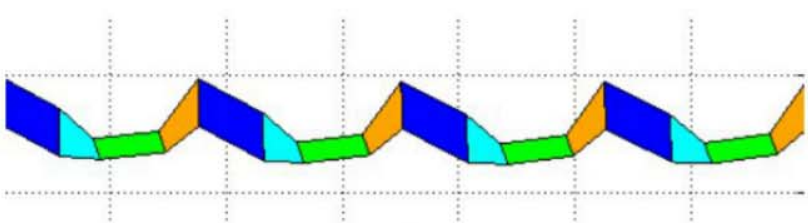

a)

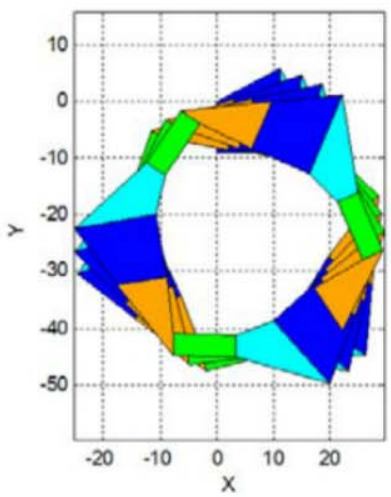

b)

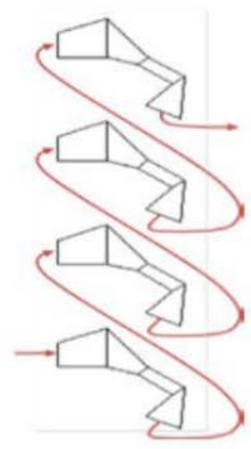

c)
Figure 6 a) Foil folded out b) Foil folded out with overlapping c) 2D continuity

geometry of only 3 turns. In Figure 5.g a larger number of turns are selected and finally the clearance is increased in Figure 5.e. The thickness of each segment in a turn can also be set individually even though the final simulation is made in 2D. This was accomplished by multiplying the resistance by a thickness factor.

A graphical user interface (GUI) was created in order to verify the geometry in terms of the dynamic parameters of the inductor. Optimizing visualization and user friendliness paid off quickly and the GUI soon became an invaluable tool. As an example the GUI was used to plot the different winding geometries in Figure 5.a-4.h. showing each parameter varied at a time.

\section{COMSOL}

In COMSOL the AC/DC module and the LiveLink to MATLAB was used to implement the optimization routine [5, 6]. COMSOL uses FEM to calculate the DC resistance of any $2 \mathrm{D}$ shape. The resistivity for each segment in a turn is utilized to model the thickness of the individual sections.

\section{A. $2 D$ mapping}

2D FEM simulations, as opposed to 3D FEM simulations, speeds up the simulation time and lower the computer resources needed.

The turns shown in Figure 7 are on purpose bendable shapes in the sense that the whole winding can be unfolded in one piece to a flat $2 \mathrm{D}$ structure by a $2 \mathrm{D}$ mapping. An example of an unfolded winding is shown in Figure 6.a. The plain 2D structure is imported to COMSOL through Matlab live-link. Unfortunately, bendable structures when unfolded can overlap them self as seen in Figure 6.b. importing an overlapping structure to COMSOL will lead to non-real result. To avoid the issue of overlap each single 2D mapped turn is taken apart and placed above each other and then imported to COMSOL. Within COMSOL each turns are mathematical linked together again by a boundary condition pair of continuity. This approach is illustrated in Figure 6.c. with 4 2D mapped turns 


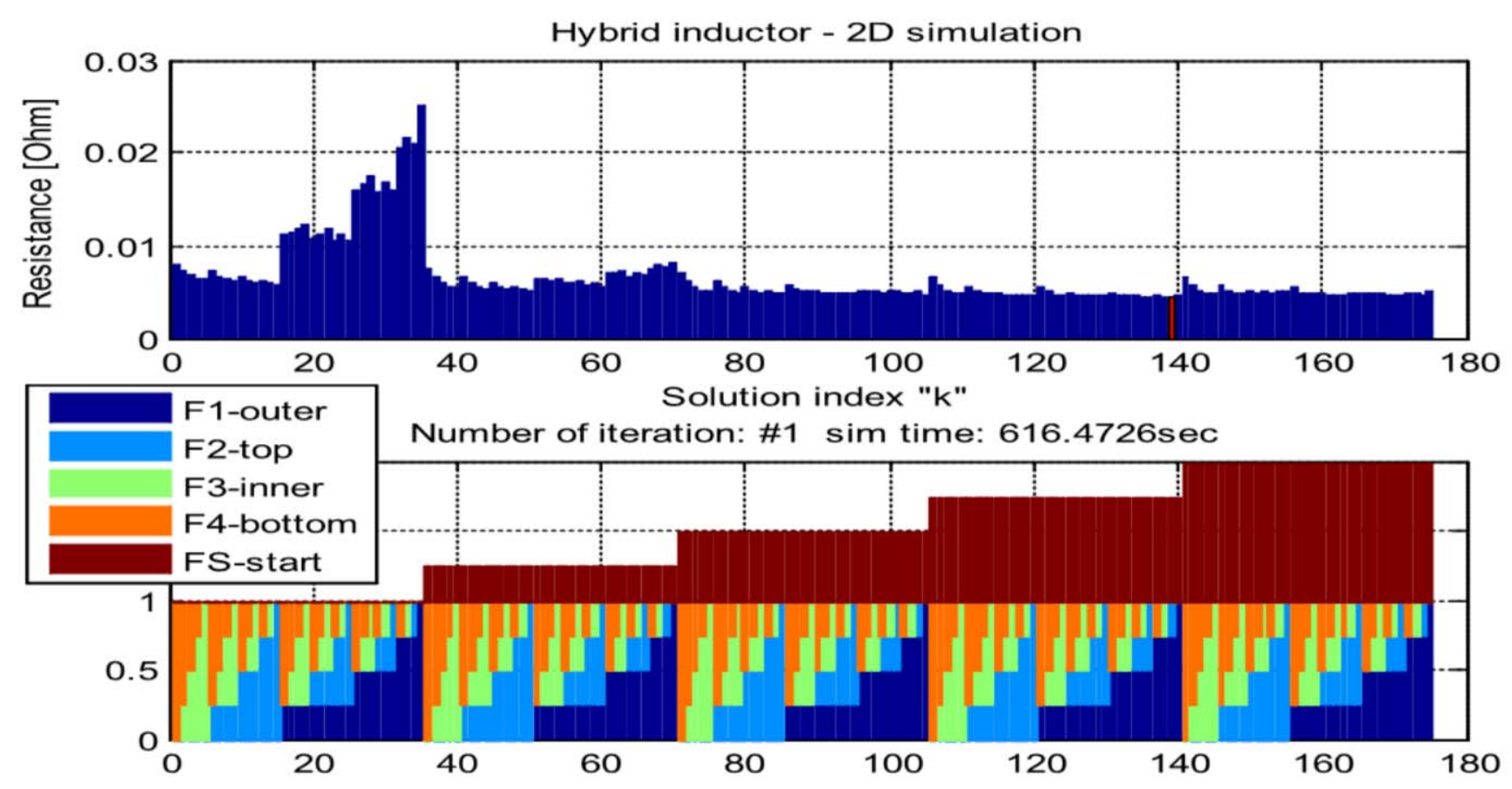

Figure 7. Illustration of optimizing routine for a single integration. The $\mathrm{x}$-axis is the combination number for the given angle span. Angle combinations are shown in the lower plot and the corresponded DC resistance is shown in the top plot.

placed above each other, the arrows indicates how the 4 shapes are linked together with boundary condition pairs of continuity.

\section{OPTIMIZATION}

Based on the FEM simulation of the DC resistance the segment angle parameters of a turn are optimized. The optimization targets the lowest DC resistance by an iterative process of narrowing down the angle span for each segment based on the parameters for previous solution. A predefined minimum and maximum value for the angle span of each segment limits the span of solution. The angle span is divided into five values. The DC resistance for the combinations of segment angels is simulated. The combination resulting in the lowest DC resistance is used to derive the set of angle spans for the next iteration. The optimization routine is ended when the preset number of iterations is reached. The lower plot in Figure 7 illustrates how the optimization routine works by stepping through the combination of angels for each segment. The resulting DC resistance is shown in the top plot of Figure 7.

\section{RESUlts}

The optimization routine was tested with a 106-2 core from Micrometals given the following parameters.

- Core height: $11.1 \mathrm{~mm}$

- Core outer radius: $26.9 \mathrm{~mm}$

- Core inner radius: $15.5 \mathrm{~mm}$

In the first test case the following winding setup was used:

- $\quad$ Segment thicknesses: F1,F2,F3 $=500 \mu \mathrm{m}$ and F4 = 70 $\mu \mathrm{m}$

- Number of turns: 10

- Clearence: $1 \mathrm{~mm}$
Resulst for three different setups are shown in TABLE I. In the "PCB $100 \%$ " the angle is $100 \%$ in the PCB trace. Since the PCB layer thickness is much thinner than the bended foil pieces it is obvious that this setup result in a high DCresistance. The "Optimal shape" is found using the optimization routine. The resistance is reduced by $32 \%$ compared to the "PCB $100 \%$ ". Only the angles in the outer and the top segment has been angled and the starting position is $20 \%$. Due to the offset of the starting position and the angle on outer segment the overall footprint of the hybrid inductor is increased. A much simpler implementation that dos not take up more space is the "Top $100 \%$ " where only the top segment is angled $100 \%$. Here an improvement off $31 \%$ is reached compared to the "PCB $100 \%$ ".

In the next test case only the number of turns was increased with a factor of 10 and the clearence was decreased with a factor of 10 . The same three setups as in case 1 was simulated. The results shown in TABLE II. indicates that the "PCB connection" is sufficient because the degree of fredom in terms of starting position and segment angles is decreased as a function of number of turns. In the second test case the

TABLE I. CASE 1

\begin{tabular}{|c|c|c|c|}
\hline & PCB 100\% & $\begin{array}{c}\text { Optimal } \\
\text { shape }\end{array}$ & Top 100\% \\
\hline SP & 0 & $20 \%$ & 0 \\
\hline F1 & 0 & $63 \%$ & 0 \\
\hline F2 & 0 & $37 \%$ & $100 \%$ \\
\hline F3 & 0 & 0 & 0 \\
\hline F4 & $100 \%$ & 0 & 0 \\
\hline DC resistance & $6.78 \mathrm{~m} \Omega$ & $4.64 \mathrm{~m} \Omega$ & $4.71 \mathrm{~m} \Omega$ \\
\hline Improvement & Ref. & $\approx 32 \%$ & $\approx 31 \%$ \\
\hline
\end{tabular}


TABLE II. CASE 2

\begin{tabular}{|c|c|c|c|}
\hline & PCB 100\% & $\begin{array}{c}\text { Optimal } \\
\text { shape }\end{array}$ & Top 100\% \\
\hline SP & 0 & 0 & 0 \\
\hline F1 & 0 & $75 \%$ & 0 \\
\hline F2 & 0 & $25 \%$ & $100 \%$ \\
\hline F3 & 0 & 0 & 0 \\
\hline F4 & $100 \%$ & 0 & 0 \\
\hline DC resistance & $435 \mathrm{~m} \Omega$ & $432 \mathrm{~m} \Omega$ & $432 \mathrm{~m} \Omega$ \\
\hline Improvement & Ref. & $\approx 0.8 \%$ & $\approx 0.8 \%$ \\
\hline
\end{tabular}

following setup was used:

- $\quad$ Segment thickness: F1,F2,F3 $=500 \mu \mathrm{m}$ and F4 $=70$ $\mu \mathrm{m}$

- Number of turns: 100

- Clearence: $0.1 \mathrm{~mm}$

In the last test case the thickness of each segment in a turn was kept constant. The result of this case could also be used in the design of a foil wounded toroidal inductor. In the third case the following setup was used:

- $\quad$ Segment thickness: F1,F2,F3,F4 $=500 \mu \mathrm{m}$

- Number of turns: 10

- Clearence: $1 \mathrm{~mm}$

The results shown in TABLE III. indicates that the "Optimal shape" is superior to both the "PCB 100\%" and the "Top $100 \%$ " in this case. The Optimal shape is $11 \%$ better than the "PCB 100\%" and 5\% better than the "Top 100\%".

\section{CONCLUSION}

The shape of a bendable foil piece for a hybrid inductor has been optimized regarding the DC-resistance. It has been found that a complex optimization isnt nessesary because the
TABLE III. CASE 3

\begin{tabular}{|c|c|c|c|}
\hline & PCB 100\% & $\begin{array}{c}\text { Optimal } \\
\text { shape }\end{array}$ & Top 100\% \\
\hline SP & 0 & $100 \%$ & 0 \\
\hline F1 & 0 & $38 \%$ & 0 \\
\hline F2 & 0 & $21 \%$ & $100 \%$ \\
\hline F3 & 0 & $0.5 \%$ & 0 \\
\hline F4 & $100 \%$ & $40.5 \%$ & 0 \\
\hline DC resistance & $2.60 \mathrm{~m} \Omega$ & $2.31 \mathrm{~m} \Omega$ & $2.46 \mathrm{~m} \Omega$ \\
\hline Improvement & Ref. & $\approx 11 \%$ & $\approx 6 \%$ \\
\hline
\end{tabular}

added advantage is small compared to a simpler implementation using a $100 \%$ top angled segment. It is also shown that as the number of turns increases an angle of $100 \%$ in the PCB is sufficient as the number of turns increases. This leads to a simplified winding assembly of the hybrid inductor.

It is observed that the optimization routine has an advantage when the thickness of each segment in a turn is constant. The conclusions in this work is only based on the DC-resistance. In future work the influence of other factors like the AC-resistance and parasitic capacitance will be taken into account. This may enable a fair comparison between the hybrid inductor vs. a conventional wire wound toroidal inductor.

\section{REFERENCES}

[1] B. Carsten, "Calculating the high frequency resistance of single and double layer toroidal windings", Micrometals,

[2] Datasheet, "WH 200 Toroid Hook winding machine", Available: www.coilwindingmachines.eu.

[3] P. Mignano, A. Mark, Huang, and C. Young, "Surface munt magnetic core winding structure", US 2004/0130428 A1, 2004.

[4] H. Schneider, T. Andersen, A. Knott, and M. A. E. Andersen. "Foil based single layer hybrid toroidal inductor", ECCE Asia Downunder, in press.

[5] "Introduction to AC/DC Module", COMSOL, Application note, 2012.

[6] "Introduction to LiveLink for MATLAB", COMSOL, Application note, 2012. 